\title{
Reduced larger von Willebrand factor multimers at dawn in OSA plasmas reflect severity of apnoeic episodes
}

\author{
Noriko Koyama*, Masanori Matsumoto*, Shinji Tamaki*, Masanori Yoshikawa*, \\ Yoshihiro Fujimura\# and Hiroshi Kimura*
}

ABSTRACT: Plasma von Willebrand factor (VWF), produced in and released from vascular endothelial cells by various stimuli including hypoxia, induces platelet aggregation under high shear stress and plays dual pivotal roles in haemostasis and thrombosis within arterioles, which are regulated by the size of vWF multimers (VWFMs).

Patients with obstructive sleep apnoea (OSA) have increased risk of thrombotic cardiovascular events, but the pathogenesis is unclear. We examined the relationship between VWF and OSA by measuring VWF antigen (VWF:Ag), VWFMs, VWF collagen binding activity (VWF:CB) and a disintegrin-like, metalloproteinase, and thrombospiondin type 1 motifs 13 . A total of 58 OSA patients were enrolled. Blood samples were collected before sleep, after sleep, and after one night of nasal continuous positive airway pressure therapy.

Based on VWFM analysis, OSA patients were classified into three groups; consistently normal VWFMs (group 1, $\mathrm{n}=29$ ), increased high molecular weight (HMW)-VWFMs at 06:00 h (group 2, $n=18$ ), and decreased or absent HMW-VWFMs at 06:00 h (group 3, $n=11$ ). Patients in group 3 had significantly worse apnoea/hypopnoea index; VWF:CB followed a similar pattern. We observed a significant decrease in platelet count between 21:00 $\mathrm{h}$ and 06:00 $\mathrm{h}$ in OSA patients, potentially associated with reduced larger VWFMs together with decreased VWF:Ag levels. Severe OSA may contribute to an arterial pro-thrombotic state.

KEYWORDS: ADAMTS13, obstructive sleep apnoea, von Willebrand factor

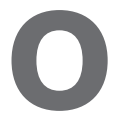
bstructive sleep apnoea (OSA) is characterised by the collapse of the upper airway and associated intermittent hypoxia during sleep [1]. OSA is associated with excessive daytime sleepiness and cardiovascular disease. Patients with OSA often suffer from obesity, hypertension, hyperlipidaemia, and impaired glucose tolerance, and OSA is an independent risk factor for cardiovascular diseases [2-4]. Consistent with this, cardiovascular risk returned to baseline in OSA patients treated with nasal continuous positive airway pressure (CPAP), whereas those with severe untreated OSA maintained a high risk [5]. Recently, some association of OSA with venous thromboembolism in regard to pulmonary embolism has been implicated [6, 7]. However, the mechanism of OSA-associated thrombosis might be multifactorial, and in fact has not been evaluated on a basis of arterial thrombosis, which is generated under high shear stress in microvasculatures, where von Willebrand factor (VWF) plays a critical role as a molecular glue that facilitates platelet aggregation or thrombi.
VWF is a macromolecular plasma protein, which cular endothelial cells, and exerts pivotal effects on both haemostasis and thrombosis. VWF assembles into unusually large VWF multimers (ULVWFMs) consisting of identical 250 kDa subunits, before its release into the circulation. Under normal circumstances, UL-VWFMs are rapidly cleaved by a specific plasma protease, ADAMTS13 (a disintegrin-like, metalloproteinase, and thrombospiondin type 1 motifs 13), under the high shear stress generated in the microvasculature; consequently, VWF circulates in the plasma as a heterogeneous family of multimers ranging in size from 500 to $15,000 \mathrm{kDa}$. UL-VWFMs play an essential role in primary haemostasis by binding platelets to denuded vascular endothelial tissue. However, in the absence of ADAMTS13 activity (ADAMTS13:AC) due to gene mutation or acquired autoantibodies, UL-VWFMs remain uncleaved and generate platelet hyperaggregation. Uncleaved UL-VWFMs lead to the formation of vast platelet thrombi, known as thrombotic is exclusively produced in and released from vas-
AFFILIATIONS

*Second Dept of Internal Medicine, Nara Medical University, and \#Dept of Blood Transfusion Medicine, Nara Medical University, Nara, Japan.

CORRESPONDENCE

H. Kimura

Second Dept of Internal Medicine Nara Medical University 840 Shijo-cho

Kashihara

Nara 634-8522 Japan E-mail: kimura@naramed-u.ac.jp

Received: Dec 032010 Accepted after revision: Jan 082012 First published online: Feb 232012 


\begin{tabular}{|c|c|c|c|}
\hline & OSA & Sleep controls & p-value \\
\hline Sex $n$ (M/F) & $58(55 / 3)$ & $25(22 / 3)$ & NS \\
\hline Blood type & & & NS \\
\hline A & 18 & 12 & \\
\hline B & 8 & 2 & \\
\hline 0 & 26 & 8 & \\
\hline$A B$ & 6 & 3 & \\
\hline Age yrs & $44.7 \pm 9.9$ & $38.3 \pm 7.1$ & $<0.01$ \\
\hline $\mathrm{BMI} \mathbf{k g} \cdot \mathrm{m}^{-2}$ & $28.2 \pm 3.7$ & $27.7 \pm 3.0$ & NS \\
\hline AHI & $50.5 \pm 22.2$ & $4.5 \pm 2.8$ & $<0.01$ \\
\hline ODI3\% & $41.6 \pm 19.9$ & $7.8 \pm 5.1$ & $<0.01$ \\
\hline Lowest $\mathrm{Sp}, \mathrm{O}_{2} \%$ & $76.0 \pm 10.0$ & $88.8 \pm 5.0$ & $<0.01$ \\
\hline Systolic blood pressure $\mathrm{mmHg}$ & $129 \pm 16$ & $122 \pm 28$ & NS \\
\hline Diastolic blood pressure $\mathrm{mmHg}$ & $82 \pm 12$ & $81 \pm 10$ & NS \\
\hline vWF:Ag levels \% at $06: 00 \mathrm{~h}$ & $103.1 \pm 61.4$ & $143.5 \pm 63.8$ & $<0.01$ \\
\hline $\begin{array}{l}\text { ADAMTS13:AC levels \% at } \\
06: 00 \mathrm{~h}\end{array}$ & $56.8 \pm 22.6$ & $61.7 \pm 20.6$ & NS \\
\hline
\end{tabular}

Data are presented as mean $\pm \mathrm{SD}$, unless otherwise stated. $\mathrm{M}$ : males: F: females; BMI: body mass index; AHI: apnoea/hypopnoea index; ODI3\%: oxygen desaturation index $\geqslant 3 \% ; \mathrm{Sp}, \mathrm{O}_{2}$ : arterial oxygen saturation measured by pulse oximetry; VWF:Ag: von Willebrand factor antigen; ADAMTS13:AC: a disintegrin-like, metalloproteinase, and thrombospiondin type 1 motifs 13 activity; NS: not significant. thrombocytopenic purpura, a life-threatening generalised disease [8-11].

It is now well established that high plasma levels of VWF antigen (VWF:Ag) are linked with an increased risk for ischaemic heart disease and ischaemic stroke [12-14]. Furthermore, the relative risks of stroke and acute myocardial infarction are higher in individuals with lower ADAMTS13:AC [14, 15]. Furthermore, hypoxia leads to increased VWF release from cultured vascular endothelial cells, both directly, by up regulating VWF expression, and indirectly via autocrine and paracrine signalling downstream of hypoxia-induced inflammatory cytokines including interleukin (IL)-6, IL-8, and tumour necrosis factor- $\alpha[16,17]$. Despite these important reports of hypoxiainduced VWF secretion, no subsequent studies have addressed the relationship between VWF and the severity of OSA [18, 19]. In particular, no studies have been performed on plasma samples obtained in chronological order relevant to the sleep cycle.

In this study, we sequentially analysed plasma VWF:Ag levels, VWFM patterns, and ADAMTS13:AC in OSA patients not only before and after sleep, but also before and after CPAP treatment. We found that the reduced larger VWFMs together with decreased VWF:Ag levels in the plasma of OSA patients taken at dawn correlate with the clinical severity of apnoeic episodes.

\section{PATIENTS, MATERIALS AND METHODS Patients}

Between February 2004 and April 2011, 284 patients received full standard diagnostic polysomnography (PSG) at Nara Medical University Hospital (Nara, Japan). Among them, 86 patients were diagnosed with normal or mild OSA (apnoea/ a)

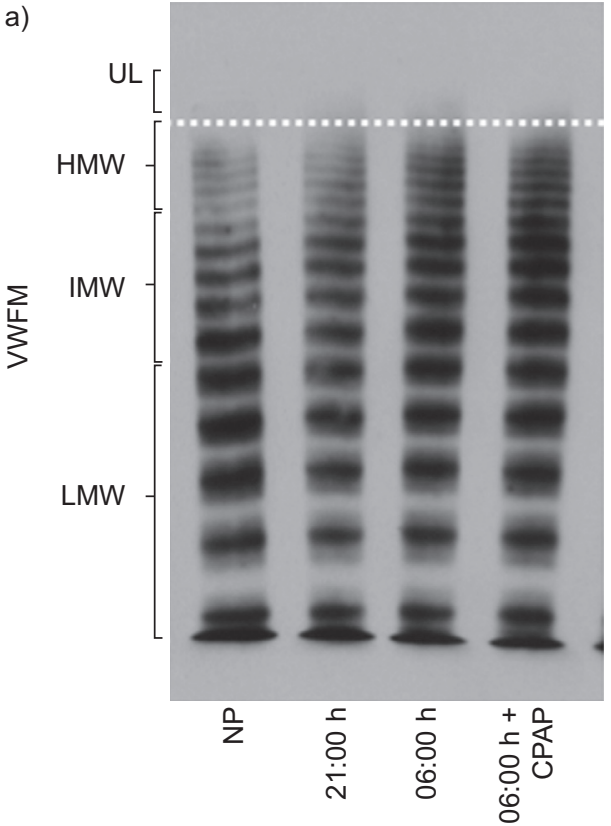

b)

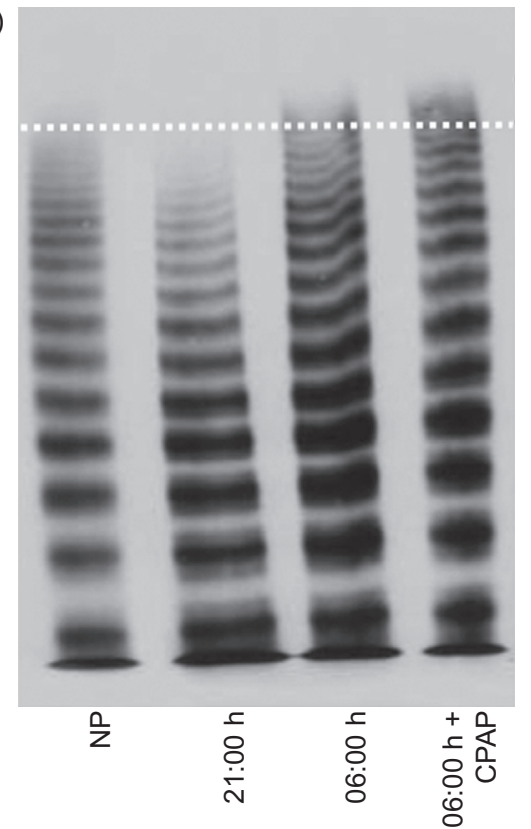

c)

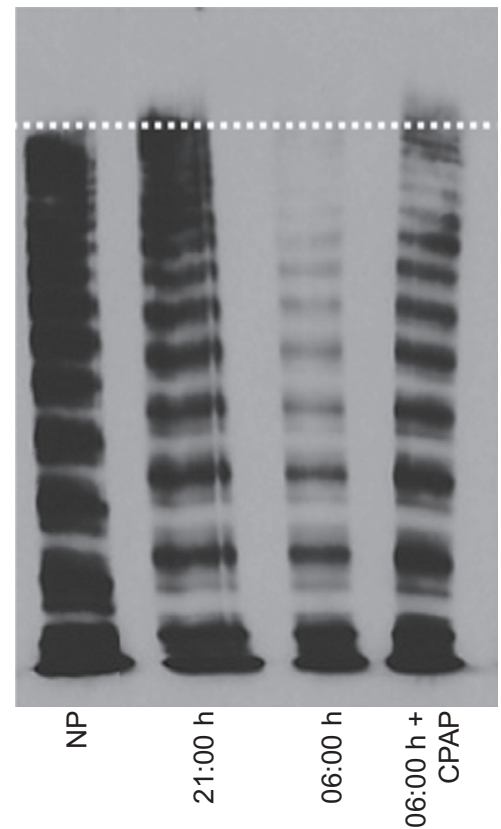

FIGURE 1. Patterns of von Willebrand factor multimers (VWFMs) corresponding to three patient groups. Obstructive sleep apnoea (OSA) patients were categorised into three groups based on the results of VWFM analysis, using sequential samples. Representative results from each group are shown. a) Group 1, patients ( $n=29)$ showed a consistently normal pattern of VWFMs. b) Group 2, patients $(n=18)$ had increased, unusually large (UL)- and high molecular weight (HMW)-VWFMs at 06:00 $h$ compared to 21:00 h. c) Group 3,patients ( $n=11$ ) had decreased UL- and HMW-VWFM at 06:00 h compared to 21:00 h. 
hypopnoea index $(\mathrm{AHI})<15)$, and 198 patients were diagnosed with moderate or severe OSA $(\mathrm{AHI} \geqslant 15)$ and received nasal CPAP therapy. Within the latter group, 140 patients with the following underlying diseases were excluded: stroke, coronary artery disease, asthma, chronic obstructive pulmonary disease, arthritis, autoimmune disease, rhinitis, and malignant diseases. The 58 remaining OSA patients were enrolled in this study; detailed clinical information for these 58 patients is shown in table S1. Written informed consent was obtained from all patients, and the study was approved by the Human Subjects Ethics Committee of Nara Medical University (No. 04-012). 25 healthy volunteers (88\% male), as shown in table 1 , that had undergone PSG studies without OSA were also enrolled and used as the sleep controls.

\section{Blood sampling}

Plasma samples were collected from OSA patients at three time points throughout the day; 21:00 $\mathrm{h}$ before PSG, at 06:00 $\mathrm{h}$ after the PSG without CPAP, and at 06:00 $\mathrm{h}$ after CPAP treatment. For the sleep control subjects, plasma samples were collected at 06:00 h. Blood was collected in plastic tubes (Becton, Dickinson and Co., Franklin Lakes, NJ, USA) containing a tenth volume of $3.8 \%$ trisodium citrate an anticoagulant, and platelet-poor plasma was prepared by centrifugation at 3,000 $\times \mathrm{g}$ for $15 \mathrm{~min}$ at $4{ }^{\circ} \mathrm{C}$. Aliquots were stored at $-80^{\circ} \mathrm{C}$ prior to use. To obtain platelet counts, blood was collected into plastic whole blood tubes with spray-coated EDTA (Becton, Dickinson and Co.) tubes containing EDTA as an anticoagulant and analysed with a Coulter counter (Beckman Coulter, Tokyo, Japan).

\section{Sleep study}

PSG was performed using a computerised polysomnography system (Alice 4; Respironics, Pittsburgh, PA, USA). Data acquisition began at 21:00 $\mathrm{h}$ and continued until 06:00 $\mathrm{h}$ the following day. Apnoea was defined as a cessation of airflow for $\geqslant 10 \mathrm{~s}$, and hypopnoea was defined as a decrease in airflow at least $50 \%$ for a minimum of $10 \mathrm{~s}$ or a clear decrease in airflow $(\geqslant 20 \%)$ followed by either oxygen desaturation $\geqslant 3 \%$ or signs of physiological arousal. The AHI was calculated as the number of apnoea/hypopnoea events per hour of total sleeping time. We also calculated the oxygen desaturation index $\geqslant 3 \%(\mathrm{ODI} 3 \%)$, defined as the number of $\geqslant 3 \%$ dips in oxygen saturation per hour of sleep.

During the night, following diagnostic PSG, patients were treated with nasal CPAP (REMstar Auto; Respironics), with PSG monitoring. Apnoeic episodes were substantially reduced or eliminated during treatment with nasal CPAP.

\section{Analyses of VWF:Ag, VWF, and VWF:CB}

Plasma VWF:Ag levels were measured by sandwich ELISA using a rabbit anti-human VWF polyclonal antiserum (DAKO, Glostrup, Denmark) [20]. The VWF:Ag level contained in $1 \mathrm{~mL}$ of pooled normal human plasma was defined as 100\%; VWF:Ag levels in the 20 healthy controls were $102 \pm 33 \%$ (mean \pm SD) [21].

VWFMs were analysed by sodium dodecyl sulphate- $1.2 \%$ agarose gel electrophoresis followed by Western blotting with luminographic detection $[22,23]$. The blots were scanned and subjected to densitometric analysis using ImageJ (National Institutes of Health (NIH), Bethesda, MD, USA). Multimers were classified as low molecular weight (LMW-VWFMs; corresponding
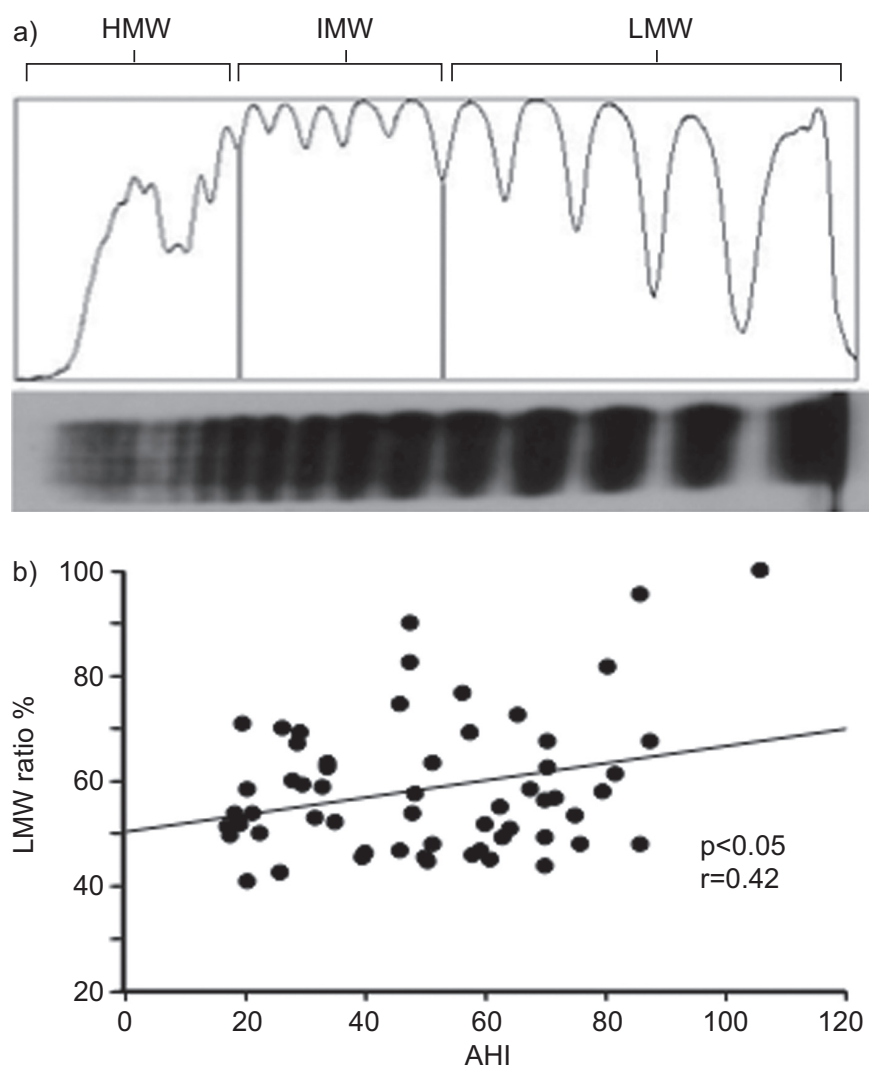

FIGURE 2. Relationship between low molecular weight (LMW) von Willebrand factor multimers (VWFMs) to total WWFMs (LMW ratio) and hypoxia. a) Quantitative analysis of VWFMs was performed by calculating the density of LMW-VWFMs relative to total $M$ density. A representative result of VWF analysis at 06:00 $\mathrm{h}$ is shown. b) The LMW ratio of obstructive sleep apnoea patients was significantly correlated to apnoea/hypopnea index (AHI). IMW: intermediate molecular weight.

to bands 1-5 in VWFM analysis), intermediate molecular weight (IMW-VWFMs; bands 6-10), and high molecular weight (HMWVWFMs; bands $\geqslant 11$ ) [24]. High molecular weight bands that were not detected in normal plasma (NP) were defined as ULVWFMs. The levels of LMW-, IMW- and HMW-VWFMs were calculated using NIH ImageJ. For quantitative analyses, we calculated the ratios of the densities of VWFMs, LMW, IMW, and HMW relative to total VWFM density. Further, multimeric VWF:Ag levels were calculated by multiplying VWF:Ag level by the LMW, IMW, and HMW ratios.

The plasma VWF collagen binding activity (VWF:CB) was measured using an enzyme immunoassay using a commercially available kit (VWF-CBA ELISA, PROGEN Biotechnik GmbH, Heidelberg, Germany) according to the manufacturer's instructions.

\section{Assay of ADAMTS13:AC}

ADAMTS13:AC was determined using a commercially available chromogenic ELISA/ACT (Kainos Co., Tokyo, Japan). The detection limit of this assay was $0.5 \%$; the values obtained from 55 healthy controls were $99.1 \pm 21.5 \%$ (mean \pm SD) [25].

\section{Statistical analysis}

Laboratory data are expressed as the mean $\pm \mathrm{SD}$. Comparisons between OSA patients and controls were analysed using the 

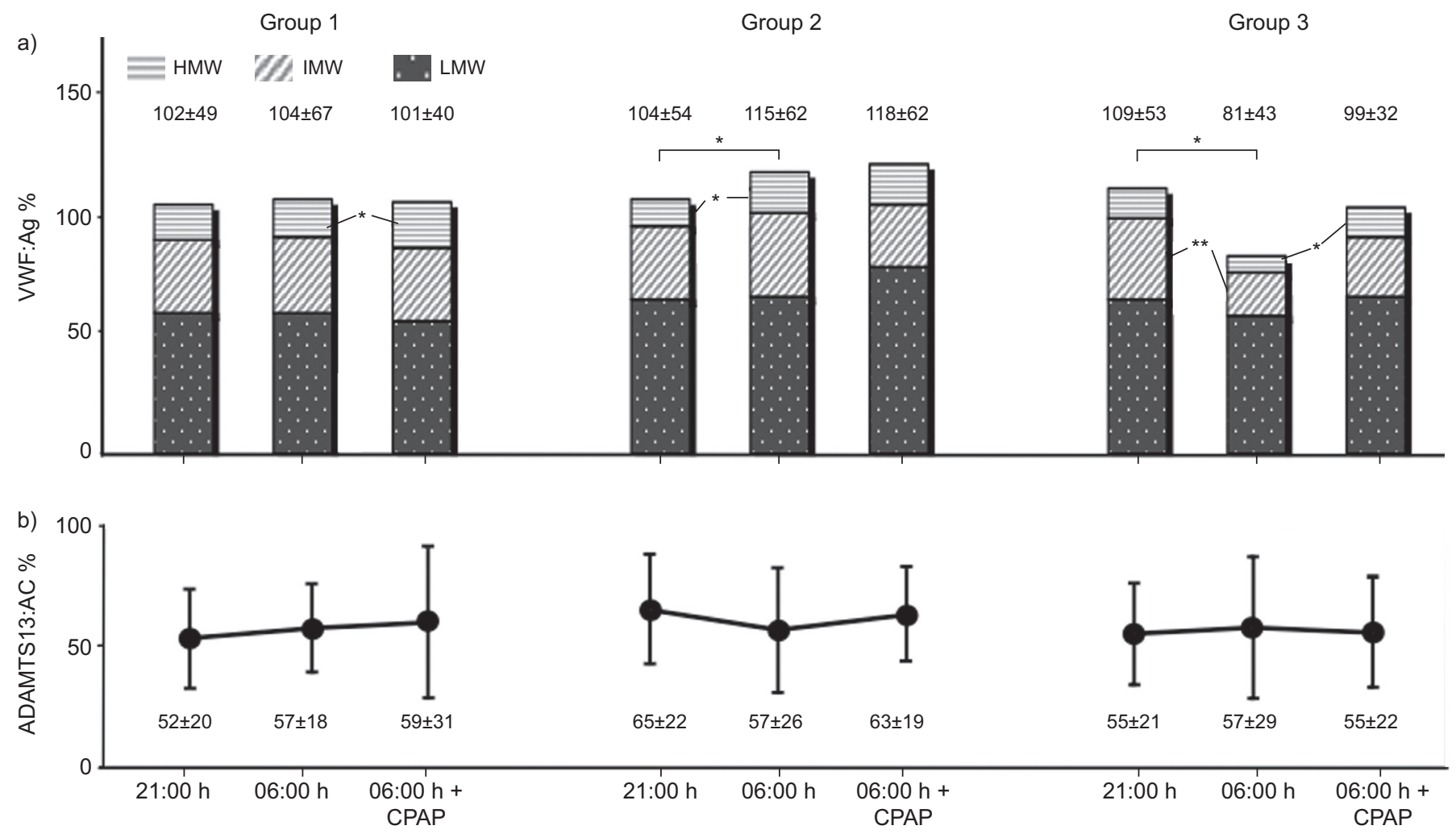

FIGURE 3. Changes in serial von Willebrand factor antigen (VWF:Ag) levels and a disintegrin-like, metalloproteinase, and thrombospiondin type 1 motifs 13 activity (ADAMTS13:AC) in groups 1-3. VWF:Ag levels were divided into high molecular weight (HMW)-, intermediate molecular weight (IMW)-, and low molecular weight (LMW)WWFM groups by multiplying the VWF:Ag level by the results of the multimeric analyses. Data are presented as mean \pm SD . Groups were first compared using the Kruskal-Wallis H-test; significantly different groups were then analysed using the Mann-Whitney U-test. *: $p<0.05 ;{ }^{* *}: p<0.01$.

Mann-Whitney U-test or Chi-square test. All comparisons among the three groups were tested for statistical significance using the Kruskal-Wallis H-test or Chi-square test, with Yates' correction for $2 \times 3$ tables; significant differences between the three groups (overall $\mathrm{p}<0.05$ ) were further analysed using the Mann-Whitney U-test or Chi-square test. All analyses were carried out using StatView (SAS Institute Inc., Cary, NC, USA). A p-value $<0.05$ was considered significant.

\section{RESULTS}

\section{Characteristics of patients with OSA and controls}

The demographics and sleep characteristics of patients with OSA and controls are shown in table 1. Patients with OSA were slightly older than the control population but were otherwise similar demographically. 18, seven, and four patients in the OSA group were being treated for hypertension, hyperlipidaemia, and diabetes mellitus, respectively, but no diabetic patients were receiving insulin therapy. Based on the PSG results, the two populations differed significantly with respect to AHI, $\mathrm{ODI} \%$, and lowest $\mathrm{Sp}, \mathrm{O}_{2} \%$.

Plasma VWF:Ag levels at 06:00 h were significantly lower in patients with OSA compared with the controls, but plasma ADAMTS13:AC at 06:00 h did not differ between these groups. Interestingly, the plasma ADAMTS13:AC at 06:00 $\mathrm{h}$ in both
OSA patients and sleep controls were lower than those of the above mentioned healthy controls $(p<0.01)$.

\section{Chronological changes of plasma VWFM patterns categorise the patients with OSA into three groups}

We analysed VWFM patterns in plasmas taken from OSA patients, obtained at 21:00 $\mathrm{h}$ and 06:00 $\mathrm{h}$ following sleep with or without CPAP. Based on these results, we categorised the patients with OSA into three groups (fig. 1). Patients in group 1 $(n=29)$ had a consistently normal pattern of VWFM, almost indistinguishable from that of the sleep controls $(n=6)$. Patients in group $2(n=18)$ exhibited reduced HMW-VWFMs at 21:00 h and persistent UL-VWFMs at 06:00 h, with or without CPAP. Patients in group $3(n=11)$ had normal VWFM patterns at 21:00 h, reduced predominantly HMW-VWFMs at 06:00 h without CPAP, and returned to a normal VWFM pattern after CPAP therapy.

The decrease in HMW-VWFMs and concomitant increase in LMW-VWFMs could reflect either enhanced proteolysis by ADAMTS13 or extensive consumption secondary to platelet aggregation. Therefore, we first calculated the ratio of LMWVWFMs to total VWFMs (LMW ratio) at 06:00 h without CPAP (fig. 2), and subsequently determined the relationship between LMW ratio and AHI. As shown in figure 2, these two parameters are significantly correlated $(p<0.05)$, suggesting that the 

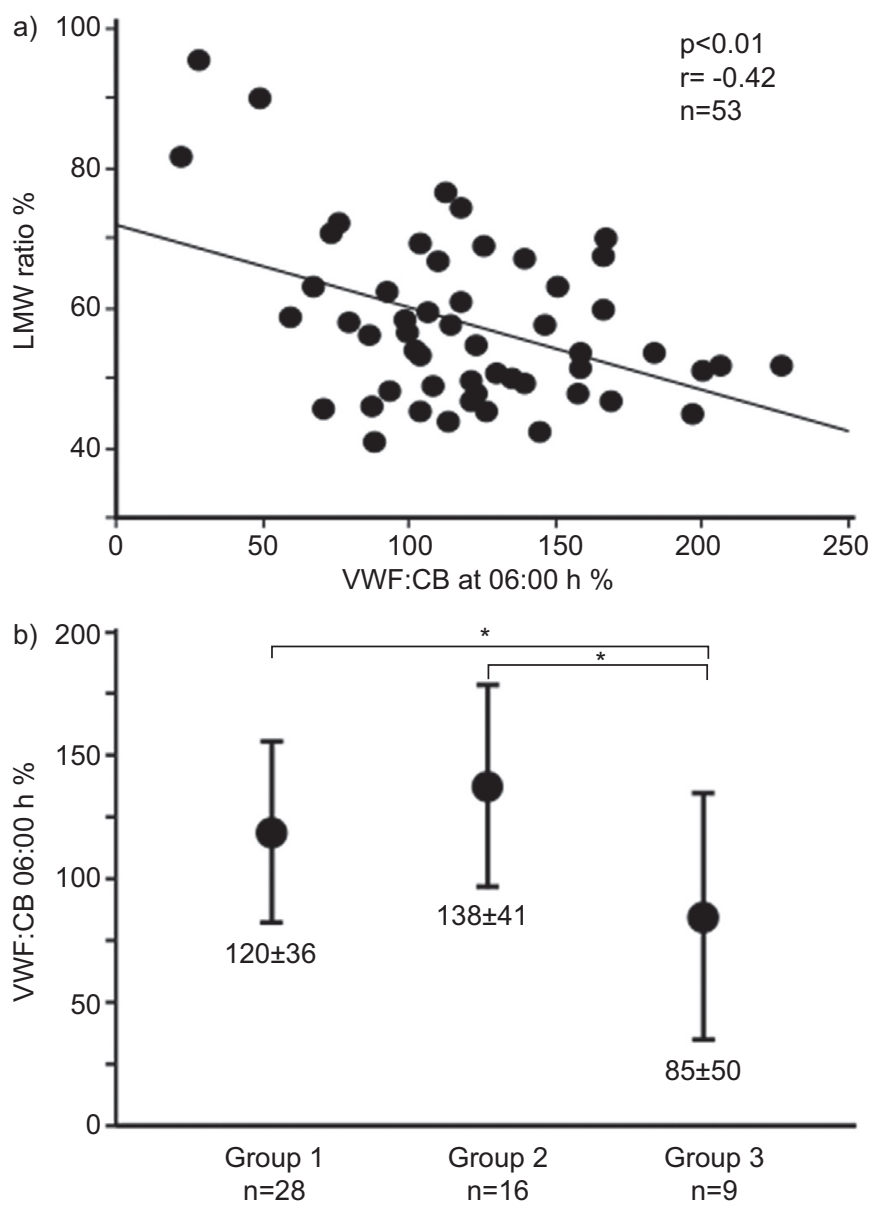

FIGURE 4. Relationship von Willebrand factor (VWF) collagen binding activity (VWF:CB) and ratio of low molecular weight (LMW)-VWF multimers (Ms) to total VWFMs (LMW ratio) and comparison of VWF:CB at 06:00 $\mathrm{h}$ in each group. VWF:CB was measured in 53 out of 58 obstructive sleep apnoea (OSA) patients. a. Significant inverse correlation between LMW ratio and VWF:CB at 06:00 $\mathrm{h}$ in OSA patients. b) VWF:CB at 06:00 $\mathrm{h}$ in group 3 was significantly lower than in groups 1 and 2. Data are presented as mean $\pm S D^{*}: p<0.05$

degree of hypoxia during apnoeic events is related to vFWMs processing and/or consumption.

\section{Chronological changes of plasma levels of VWF:Ag, VWFM ratio, and ADAMTS13:AC in three patient groups with OSA}

Plasma levels of VWF:Ag at 21:00 h, 06:00 h without CPAP, and 06:00 $\mathrm{h}$ with CPAP were determined in all three groups of OSA patients. As shown in figure 3, plasma VWF:Ag levels were almost unchanged in group 1 patients, but significantly increased between 21:00 $\mathrm{h}$ and 06:00 $\mathrm{h}$ in group 2 patients. Notably, VWF:Ag levels remarkably decreased between 21:00 h and 06:00 $\mathrm{h}$ in group 3.

We then determined levels of HMW, IMW, and LMW in all three groups. In group 1, HMW-VWFM showed a slight increase at 06:00 $\mathrm{h}$ with $\mathrm{CPAP}$, relative to 06:00 $\mathrm{h}$ without CPAP. In group 2, HMW-VWFMs significantly increased at 06:00 $\mathrm{h}$ compared to 21:00 $\mathrm{h}$ confirming the results of the VWFM analysis used for defining groups $1-3$. Consistent with this, in group 3, the IMW-VWFMs at 06:00 h was significantly lower than that at 21:00 $\mathrm{h}$; CPAP treatment reversibly increased the HMW-VWFM at 06:00 h, in accordance with the increase in plasma VWF:Ag level.

In contrast, no change in the plasma ADAMTS13:AC was seen at 21:00 $\mathrm{h}, 06: 00 \mathrm{~h}$, or 06:00 $\mathrm{h}$ with CPAP in any of the three groups. These data argue that consumption of the HMWVWFMs occurred overnight in OSA patients.

\section{Plasma levels of VWF:CB activity}

We observed dynamic chronological changes in plasma VWF: Ag levels and VWFM patterns in our subjects, especially in group 3. VWF:CB represents a biological function of VWF, in which HMW-VWFM adheres to collagen with a higher binding affinity than IMW- or LMW-VWFM. In this study, we were able to examine plasma VWF:CB levels in 53 out of 58 OSA patients. As expected, plasma levels of VWF:CB at 06:00 h without CPAP were inversely correlated with the LMW ratio $(\mathrm{p}<0.01)$, as shown in figure 4. Furthermore, as shown in figure 4, plasma levels of VWF:CB at 06:00 h was significantly lower in group $3(85 \pm 50 \%)$ than in either group $1(120 \pm 36 \%)$ or group $2(138 \pm 41 \%)$. These results argue that structurally and functionally impaired VWFMs were present at 06:00 h in group 3 patients.

\section{Decreased platelet counts at dawn in the untreated patients with OSA}

A pair of platelet counts at 21:00 h and 06:00 h without CPAP was determined in 31 of the 58 OSA patients and in six of the 25 sleep controls, all of whom were involved in the later phase of this study. To correct for a possible hydration effect during sleep, we calculated the ratio of platelet count to haematocrit. The ratios in sleep controls did not exhibit significant changes between 21:00 $\mathrm{h}$ and 06:00 $\mathrm{h}$ (fig. 5), whereas they were lower at 06:00 $\mathrm{h}$ in untreated OSA patients $(\mathrm{p}<0.01)$ (fig. 5). However, none of the patients who received CPAP treatment developed overt clinical signs of thrombotic complications. These results suggest that platelet consumption, to a lesser extent, might occur during sleep without distinct thrombotic symptoms in untreated OSA patients.

\section{Patient characteristics of groups 1, 2, and 3}

Table 2 summarises the demographic and measured parameters of OSA patients categorised into groups 1-3. These three groups did not differ demographically, but AHI was significantly higher in group 3 than in groups 1 and 2. ODI3\% in group 3 was also significantly higher than in group 1 . These results unambiguously indicate that patients in group 3, who exhibit lower levels of large VWFM at 06:00 h represent the highest severity of OSA among the three groups.

Consistent with these results, decreased plasma levels of VWF:Ag in the two different time intervals (06:00 $\mathrm{h}$ and 21:00 h) was remarkable in group 3 , in comparison to those in groups 1 and 2. Interestingly, the differences of LMW ratio in the two times (06:00 $\mathrm{h}$ and 21:00 h) was significantly higher in group 3 than those of groups 1 or 2 . These results indicated that decreased VWF:Ag at 06:00 h was caused primarily by the reduction in larger VWFMs. Alternatively, no significant change in ADAMTS13:AC between the two times (06:00 $\mathrm{h}$ and 21:00 h) was observed in group 3, whereas such a change was observed in groups 1 and 2, leaving the physiological relevance unaddressed. 

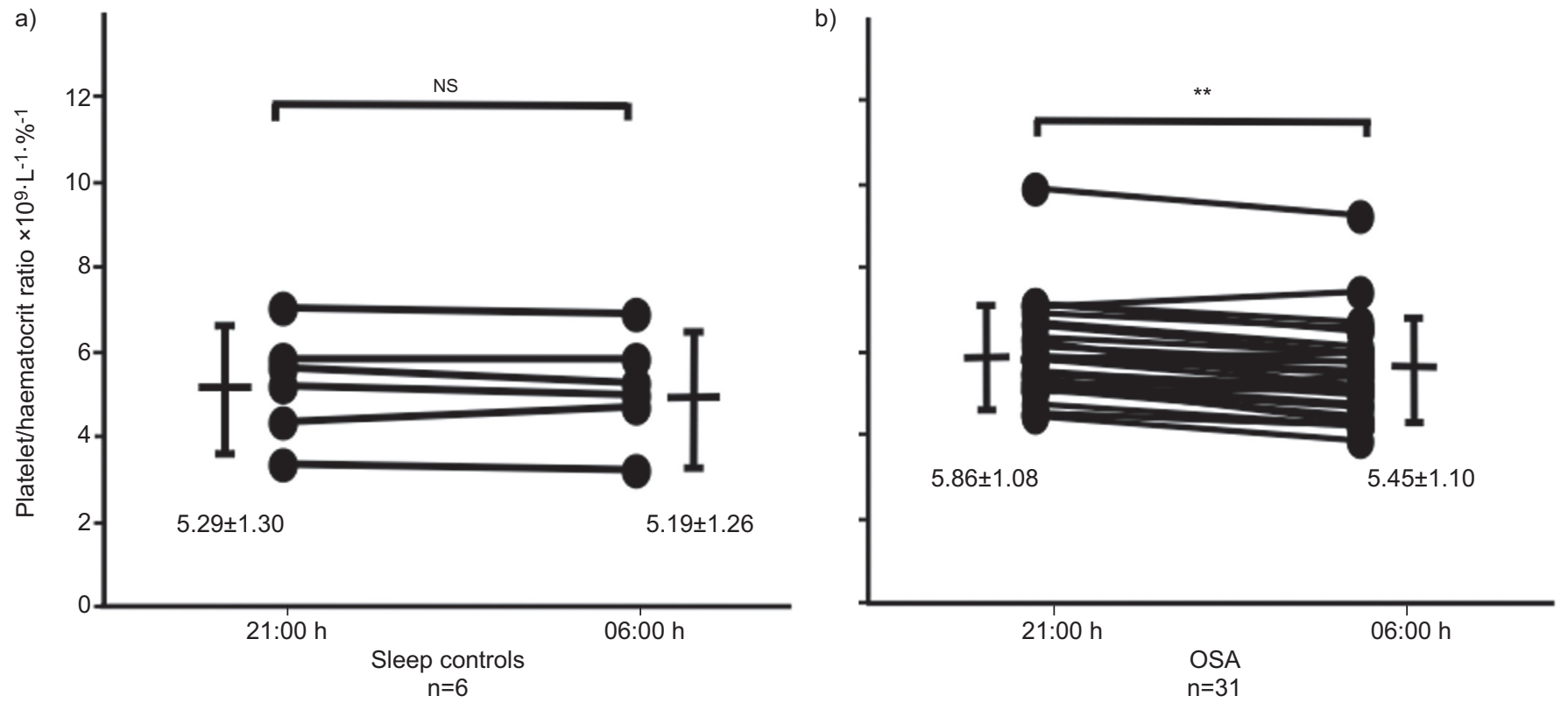

FIGURE 5. Overnight platelet counts to haematocrit ratios decreased in patients with obstructive sleep apnoea (OSA). Platelet counts were normalised to the patient's haematocrit to control for differences in hydration status. Ratios of platelet count to haematocrit were obtained at 21:00 h and 06:00 h in a) six sleep controls and in b) 31 OSA patients, both without CPAP treatment. In the sleep controls, the ratios did not change between time points. In the OSA patients, the ratio exhibited significant changes between time points. Data are presented as mean \pm SD. NS: nonsignificant. **: $p<0.01$

\section{Relationship of AHI and groups 1-3 of VWFM patterns in OSA patients}

$\mathrm{AHI}$ is an excellent means of showing OSA severity, here we have used it to categorise three groups: moderate $(15 \leqslant \mathrm{AHI}<30)$, severe $(30 \leqslant A H I<60)$, and extremely severe $(A H I \geqslant 60)$. As shown in table 3, OSA patients with group 1 and 2 consisted of those with variable AHI levels. Notably, none of the OSA patients within group 3 had an AHI $15 \sim<30$, and they uniformly

\section{TABLE 2 Characteristics and different parameter between 21:00 $\mathrm{h}$ and 06:00 $\mathrm{h}$ of patients with obstructive sleep apnoea (OSA) in groups 1-3}

Group

$+2$

Sex $M / F$

Blood type

A

B

O

$\mathrm{AB}$

Age yrs

AHI

ODI3\%

Differences in time intervals 06:00 and 21:00 h

WWF:Ag \%

LMW ratio \%

ADAMTS13:AC \%

$\mathrm{Plt} / \mathrm{Ht} \times 10^{9} \cdot \mathrm{L}^{-1} \cdot \%^{-1}$
28/1

$18 / 0$

1

7

4

14

4

$46.0 \pm 9.6$

$43.1 \pm 20.0$

$35.7 \pm 18.2$

$2.1 \pm 34.8$

$-0.27 \pm 5.24$

$4.4 \pm 13.1$

$-0.045 \pm 0.036(n=15)$

4

3

$42.9 \pm 9.7$

$51.4 \pm 19.6$

$44.1+19.3$

$10.8 \pm 22.0$

$-4.46 \pm 8.69$

$-8.5 \pm 25.9$

$-0.034 \pm 0.038(n=10)$
Overall p-value

Data are presented as $n$ or mean \pm SD, unless otherwise stated. M: males; F: females; $A H I$ : apnoea/hypopnea index, ODI3\%: oxygen desaturation index $\geqslant 3 \%$; WF:Ag : von Willebrand factor antigen; LMW ratio: the ratio of low molecular weight-VWFMs to total VWFMs; ADAMTS13:AC: a disintegrin-like, metalloproteinase, and thrombospiondin type 1 motifs 13 activity; PIt/Ht: platelet count to haematocrit ratio. NS: not significant. *: $p<0.05$ between groups 1,2 and 3 ; ${ }^{*}$ : $p<0.01$ between groups 1 and $3 ;{ }^{\bullet}: p<0.01$ between groups 1,2 and $3 ;^{+}: p<0.05$ between group 1 and 2 . 
TABLE 3 Characteristics and thrombotic parameters of patients classified with apnoea/hypopnoea index (AHI)

\begin{tabular}{|c|c|c|c|c|}
\hline & $15 \leqslant A H I<30$ & $30 \leqslant \mathrm{AHI}<60$ & $A H I \geqslant 60$ & Overall $p$-value \\
\hline Patients $\mathrm{n}$ & 15 & 22 & 21 & \\
\hline Age yr & $43.7 \pm 12.0$ & $42.9 \pm 9.7$ & $44.2 \pm 11.3$ & NS \\
\hline ODI3\% & $19.2 \pm 4.9$ & $36.2 \pm 10.9$ & $63.3 \pm 9.4$ & $<0.01^{\star \star}$ \\
\hline \multicolumn{5}{|l|}{ VWFM group } \\
\hline 3 & 0 & $4(18)$ & $7(33)$ & $<0.05^{\#}$ \\
\hline VWF:Ag at $06: 00 \mathrm{~h} \%$ & $98.5 \pm 49.1$ & $98.5 \pm 55.7$ & $111.3 \pm 75.5$ & NS \\
\hline ADAMTS13:AC at 06:00 $\mathrm{h} \%$ & $58.1 \pm 20.2$ & $55.2 \pm 21.9$ & $57.6 \pm 25.6$ & NS \\
\hline VWF:CB at 06:00 h U. $\mathrm{mL}^{-1}$ & $1.29 \pm 0.39(n=13)$ & $1.23 \pm 0.50(n=19)$ & $1.09 \pm 0.38(n=19)$ & NS \\
\hline $\mathrm{PIt} / \mathrm{Ht}$ at $06: 00 \mathrm{~h} \times 10^{9} \cdot \mathrm{L}^{-1} \cdot \%^{-1}$ & $0.526 \pm 0.093(n=10)$ & $0.549 \pm 0.138(n=13)$ & $0.561 \pm 0.087(n=8)$ & NS \\
\hline
\end{tabular}

had $\mathrm{AHI} \geqslant 30$ and more predominantly with $\mathrm{AHI} \geqslant 60$. The incident for group 1 patients was lower in $\mathrm{AHI}$ groups of $30 \leqslant \mathrm{AHI}<60$ and $\mathrm{AHI} \geqslant 60$ than those of $15 \leqslant \mathrm{AHI}<30(\mathrm{p}<0.05)$. In contrast, the incident for group 3 was higher in $\mathrm{AHI} \geqslant 60$ than those of $15 \leqslant \mathrm{AHI}<30$ ( $\mathrm{p}<0.05$ ). No significant relationship between AHI score and each parameter such as VWF, ADAMTS13, or platelet count was found.

\section{DISCUSSION}

Plasma VWF:Ag levels increase after the age of $40 \mathrm{yrs}$ in normal individuals; by the age of 60 yrs they can have reached $\sim 120-140 \%$ of the healthy normal baseline [26]. The mean age of OSA patients enrolled in this study was $44.7 \mathrm{yrs}$, whereas that of control subjects was 38.3 yrs. However, the plasma VWF:Ag levels collected at 06:00 h were significantly lower for OSA patients than for control subjects (table 1). In contrast, plasma ADAMTS13 activity decreases after the age of $40 \mathrm{yrs}$ in normal individuals [27]. Among our study patients and controls, plasma ADAMTS13:AC was lower than in healthy controls aged between 20-40 yrs $(p<0.01)$, indicating that these two groups did not significantly differ (table 1).

Given the observed differences in VWF:Ag levels between OSA patients and control subjects, we analysed VWFM patterns chronologically at three time points: at 21:00 h and at 06:00 h either with or without overnight CPAP treatment. As expected, a majority of OSA patients $(29(50 \%)$ out of 58$)$ had consistently normal VWFM patterns, categorised as group 1. Two smaller groups of patients had increased UL- and HMW-VWFM (18 (31\%) out of 58 ) or decreased UL- and HMW-VWFM (11 (19\%) out of 58) at 06:00 h; these were categorised as group 2 or group 3 , respectively. The ratio of LMW-VWFM to total VWFM, termed the LMW ratio, is a determination of the relative amount of degraded VWFM; in our study population, the LMW ratio correlated significantly with the AHI.

The increased LMW ratio seen in OSA patients could arise from reduced production of VWF by vascular endothelial cells, increased clearance of HMW-VWFM from the circulation, or consumption during thrombosis. However, in vitro studies have clearly shown that VWF expression by cultured vascular endothelial cells is increased under conditions of hypoxia; it is unlikely that patients with OSA, a condition of intermittent hypoxia, would exhibit decreased expression of VWF overnight [17]. Additionally, no differences were seen in the plasma ADAMTS13:AC in any group at any time-point, suggesting that enhanced proteolysis of HMW-VWFM was not occurring. Therefore, we hypothesised that the elevated LMW ratio seen in our OSA patients was likely to be due to an enhanced degradation or consumption of HMW-VWFM.

The cause of thrombotic complications in OSA patients might be multifactorial, but in this study we have clearly indicated that VWF appears to play an essential role in the thrombogenesis in a certain population categorised as group 3 . Although the mechanism is not yet fully elucidated, the high VWFMs released upon hypoxia from vascular endothelial cells is a most plausible factor. Thus, severe OSA could be a risk factor for both arterial and venous thrombosis as described in the introduction.

To better understand whether some degree of thrombosis was occurring overnight in untreated OSA patients, we determined platelet counts in 31 out of 58 patients; we observed a significant decrease in platelet count between 21:00 h and 06:00 $\mathrm{h}$. This decrease was associated with reductions in both the plasma VWF:Ag levels and HMW-VWFM in group 3. Quantitative analyses of VWFMs in group 3 showed that levels of HMWVWFMs increased significantly after CPAP treatment, compared with measurements taken at 06:00 h without CPAP. This is consistent with low-level consumption of UL- and HMWVWFMs by microvascular thrombus formation and/or platelet aggregation during sleep in OSA patients; CPAP therapy might reduce such consumption. However, no patients have developed overt clinical signs of thromboembolic complications; therefore, we prefer to use the term "pre-clinical platelet consumption" 
to describe this phenomenon. This may represent a baseline pro-thrombotic state in OSA patients that can be corrected by CPAP therapy.

In this study, the chronological analyses have unanimously indicated that reduced large VWFMs in plasmas at dawn reflect the clinical severity of apnoea in OSA patients. The results obtained by VWFM analysis were solid, but the procedure was time consuming and requires a high technical skill to perform. A reliable high-throughput method would be necessary for routine clinical use. In this regard, the assay for VWF:CB is a promising candidate for such a method, because HMW-VWFM adheres to collagen with a higher binding affinity than IMW- or LMW-VWFM. Our results indicated that VWF:CB at 06:00 h correlated well with VWFM patterns, and was consistent with earlier assignment of subjects to groups 1-3. Thus, through this study we have provided the first convincing evidence that VWF at dawn in group 3 was impaired not only structurally but also functionally, presumably due to hypoxia-induced release and consumption of VWF. This process might also involve platelet aggregation and consumption, even though the patients were asymptomatic. Thus, large scale studies, together with chronological measurements of platelet counts and VWF:CB, would be the focus in the following studies.

\section{SUPPORT STATEMENT}

This study was partly supported by research grants to the Respiratory Failure Research Group and Coagulation Abnormalities Research Group from the Ministry of Health, Labour and Welfare and from the Ministry of Education, Culture, Sports, Science and Technology of Japan.

\section{STATEMENT OF INTEREST}

None declared.

\section{ACKNOWLEDGEMENTS}

We would like to thank K. Makinodan, A. Fukuoka, and M. Yamauchi (Second Dept of Internal Medicine, Nara Medical University, Nara, Japan) for collecting blood samples and A. Isonishi (Dept of Blood Transfusion Medicine, Nara Medical University) for her excellent technical assistance. We would also like to thank M. Uemura (Third Dept of Internal Medicine, Nara Medical University) for his critical reading of this manuscript.

\section{REFERENCES}

1 Malhotra A, White DP. Obstructive sleep apnoea. Lancet 2002; 360: 237-245.

2 Mooe T, Rabben T, Wiklund U, et al. Sleep-disordered breathing in men with coronary artery disease. Chest 1996; 109: 659-663.

3 Shahar E, Whitney CW, Redline S, et al. Sleep-disordered breathing and cardiovascular disease: cross-sectional results of the Sleep Heart Health Study. Am J Respir Crit Care Med 2001; 163: 19-25.

4 Redline S, Yenokyan G, Gottlieb DJ, et al. Obstructive sleep apneahypopnea and incident stroke: the sleep heart health study. Am J Respir Crit Care Med 2010; 182: 269-277.

5 Marin JM, Carrizo SJ, Vicente E, et al. Long-term cardiovascular outcomes in men with obstructive sleep apnoea-hypopnoea with or without treatment with continuous positive airway pressure: an observational study. Lancet 2005; 365: 1046-1053.

6 Epstein MD, Segal LN, Ibrahim SM, et al. Snoring and risk of obstructive sleep apnea in patients with pulmonary embolism. Sleep 2010; 33: 1069-1074.

7 Bosanquet JP, Bade BC, Zia MF, et al. Patients with venous thromboembolism appear to have higher prevalence of obstructive sleep apnea than the general population. Clin Appl Thromb Hemost 2011; 17: E119-E124.

8 Ruggeri ZM. Von Willebrand factor, platelets and endothelial cell interactions. J Thromb Haemost 2003; 1: 1335-1342.

9 Sadler JE, Moake JL, Miyata T, et al. Recent advances in thrombotic thrombocytopenic purpura. Hematology Am Soc Hematol Educ Program, 2004: 407-423.

10 Moake JL, Rudy CK, Troll JH, et al. Unusually large plasma factor VIII:von Willebrand factor multimers in chronic relapsing thrombotic thrombocytopenic purpura. N Engl J Med 1982; 307: 1432-1435.

11 Levy GG, Nichols WC, Lian EC, et al. Mutations in a member of the ADAMTS gene family cause thrombotic thrombocytopenic purpura. Nature 2001; 413: 488-494.

12 Thompson SG, Kienast J, Pyke SD, et al. Hemostatic factors and the risk of myocardial infarction or sudden death in patients with angina pectoris. European Concerted Action on Thrombosis and Disabilities Angina Pectoris Study Group. N Engl J Med 1995; 332: 635-641.

13 Chion CK, Doggen CJ, Crawley JT, et al. ADAMTS13 and von Willebrand factor and the risk of myocardial infarction in men. Blood 2007; 109: 1998-2000.

14 Bongers TN, de Maat MP, van Goor ML, et al. High von Willebrand factor levels increase the risk of first ischemic stroke: influence of ADAMTS13, inflammation, and genetic variability. Stroke 2006; 37: 2672-2677.

15 Kaikita K, Soejima K, Matsukawa M, et al. Reduced von Willebrand factor-cleaving protease (ADAMTS13) activity in acute myocardial infarction. J Thromb Haemost 2006; 4: 2490-2493.

16 Wilkie ME, Stevens CR, Cunningham J, et al. Hypoxia-induced von Willebrand factor release is blocked by verapamil. Miner Electrolyte Metab 1992; 18: 141-144.

17 Pinsky DJ, Naka Y, Liao H, et al. Hypoxia-induced exocytosis of endothelial cell Weibel-Palade bodies. A mechanism for rapid neutrophil recruitment after cardiac preservation. J Clin Invest 1996; 97: 493-500.

18 von Känel R, Loredo JS, Ancoli-Israel S, et al. Association between sleep apnea severity and blood coagulability: treatment effects of nasal continuous positive airway pressure. Sleep Breath 2006; 10: 139-146.

19 Robinson GV, Pepperell JC, Segal HC, et al. Circulating cardiovascular risk factors in obstructive sleep apnoea: data from randomised controlled trials. Thorax 2004; 59: 777-782.

20 Bartlett A, Dormandy KM, Hawkey CM, et al. Factor-VIII-related antigen: measurement by enzyme immunoassay. Br Med J 1976; 1: 994-996.

21 Matsumoto $M$, Kawaguchi S, Ishizashi $\mathrm{H}$, et al. Platelets treated with ticlopidine are less reactive to unusually large von Willebrand factor multimers than are those treated with aspirin under high shear stress. Pathophysiol Haemost Thromb 2005; 34: 35-40.

22 Ruggeri ZM, Zimmerman TS. Variant von Willebrand's disease: characterization of two subtypes by analysis of multimeric composition of factor VIII/von Willebrand factor in plasma and platelets. J Clin Invest 1980; 65: 1318-1325.

23 Budde U, Schneppenheim R, Plendl H, et al. Luminographic detection of von Willebrand factor multimers in agarose gels and on nitrocellulose membranes. Thromb Haemost 1990; 63: 312-315.

24 Veyradier A, Nishikubo T, Humbert M, et al. Improvement of von Willebrand factor proteolysis after prostacyclin infusion in severe pulmonary arterial hypertension. Circulation 2000; 102: 2460-2462.

25 Kato S, Matsumoto M, Matsuyama T, et al. Novel monoclonal antibody-based enzyme immunoassay for determining plasma levels of ADAMTS13 activity. Transfusion 2006; 46: 1444-1452.

26 Conlan MG, Folsom AR, Finch A, et al. Associations of factor VIII and von Willebrand factor with age, race, sex, and risk factors for atherosclerosis. The Atherosclerosis Risk in Communities (ARIC) Study. Thromb Haemost 1993; 70: 380-385.

27 Kokame K, Nobe Y, Kokubo Y, et al. FRETS-VWF73, a first fluorogenic substrate for ADAMTS13 assay. Br J Haematol 2005; 129: 93-100. 